\title{
TRANSITIONS PHOTOINDUITES ULTRARAPIDES : UN AXE ÉMERGENT POUR LA PHOTONIQUE
}

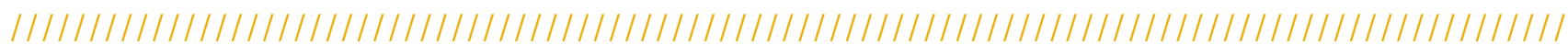

\section{Éric COLLET ${ }^{1}$}

${ }^{1}$ Univ Rennes, CNRS, IPR (Institut de Physique de Rennes) - UMR 6251, F-35000 Rennes, France

*eric.collet@univ-rennes1.fr

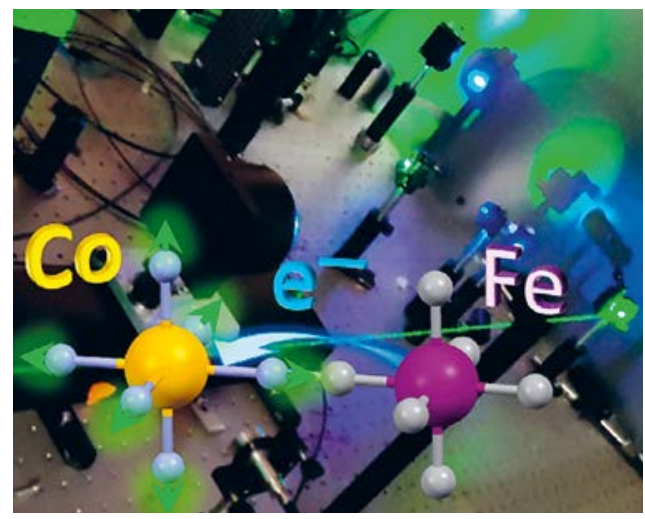

Certains matériaux changent de propriétés physiques sous lumière. Comprendre les mécanismes élémentaires pilotant ces transitions de phases photoinduites, où l'excitation électronique réorganise la structure moléculaire et induit de nouvelles fonctions, nécessite d'observer la matière se transformer à l'échelle de temps de 10 femtosecondes à l'aide d'expériences pompe-sonde de spectroscopie optique au laboratoire et de spectroscopie de rayons $X$ sur X-FEL.

Article publié en accès libre sous les conditions définies par la licence Creative Commons Attribution License CC-BY (https://creativecommons.org/licenses/by/4.0), qui autorise sans restrictions l'utilisation, la diffusion, et la reproduction sur quelque support que ce soit, sous réserve de citation correcte de la publication originale.

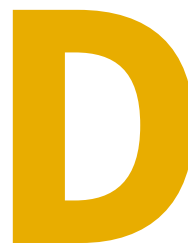

ifférents types de matériaux voient leurs propriétés physiques modifiées par la lumière. L'étude de transitions de phases photoinduites en science des matériaux ouvre une nouvelle voie pour la photonique, car la lumière permet de contrôler diverses fonctions (magnétisme, conductivité optique ou électrique, ferroélectricité,...) sur des échelles de temps ultra-rapides [1-4]. C'est un axe de recherche très en amont pour la fabrication de futurs composants permettant de moduler la transmission de signaux optiques ou de les convertir en signaux électroniques [5]. Ces transformations de matériaux peuvent être induites par des excitations lumineuses dans diverses gammes spectrales (UV, visible, infrarouge et même THz).
Considérons dans une approche simplifiée que la couleur d'un matériau est liée aux transitions électroniques entre niveaux d'énergie, à l'intensité de ces transitions et à l'occupation de ces niveaux d'énergie par les électrons. Dans une molécule, les écarts entre ces niveaux d'énergie sont gouvernés par sa structure atomique. La figure 1 montre, de façon très schématique, une molécule à transition de spin à base d'ion $\mathrm{Fe}^{2+}$ qui change de couleur entre un état bas spin (LS jaune) et haut spin (HS noir). Dans l'état LS les 6 électrons du $\mathrm{Fe}^{2+}$ dans le champ du ligand $\mathrm{L}$ occupent les niveaux d'énergie $t_{2 g}$ et ils sont appariés. Le système est diamagnétique. Dans l'état HS des électrons se répartissent sur les niveaux $t_{2 g}$ et $e_{g}$, et ne sont plus appariés. Le système est dans un état paramagnétique. Le peuplement des niveaux d'énergie $e_{g}$ diminue la force des liaisons entre le Fe et le ligand L, et ces liaisons Fe-L s'allongent durant le processus de conversion entre état LS et HS. Dans l'état LS, la transition électronique $t_{2 g} \rightarrow L$ (vers le ligand) correspond à l'absorption d'un photon bleu $(400 \mathrm{~nm})$. Le système absorbe peu au-dessus de $400 \mathrm{~nm}$ et le cristal est donc jaune. Dans l'état HS, les changements de longueurs de liaisons $\mathrm{Fe}-\mathrm{N}$ modifient les niveaux d'énergie et la transition $\mathrm{t}_{2 \mathrm{~g}} \rightarrow \mathrm{L}$ correspond au vert-jaune. De plus, les états $e_{\mathrm{g}}$ étant peuplés, une nouvelle transition $\mathrm{e}_{\mathrm{g}} \rightarrow \mathrm{L}$ apparait dans le rouge. Le cristal est alors noir car il absorbe différentes longueurs d'onde visibles. Les deux états possédant des entropies et volumes différents, il est possible d'induire ce changement d'état de spin en jouant sur la température ou la pression. Mais plus intéressant encore, il est possible de changer d'état 
de spin en excitant ce système avec la lumière. C'est l'effet LIESST (light-induced excited spin state trapping) [1-4]. En science des matériaux, la compréhension et le contrôle des processus microscopiques élémentaires pilotant ces transformations photoinduites jouent un rôle central. Observer les réorganisation électroniques et atomiques associées nécessite d'étudier la dynamique de ces transformations avec une résolution temporelle inférieure à 100 fs.

\section{EXPÉRIENCES POMPE-SONDE FEMTOSECONDE DE SPECTROSCOPIES OPTIQUE ET RAYONS X}

En discothèque, il est facile de décomposer les mouvements de danseurs à laide des flashs de lumière des stroboscopes. De la même façon, il est possible de suivre les dynamiques des électrons et des atomes à l'aide d'impulsions laser ultrabrèves en utilisant des techniques dites "pompe - sonde" (Fig. 2a). Une première impulsion laser optique de "pompe" vient exciter le système et déclenche sa transformation et une seconde impulsion de "sonde" vient mesurer l'état du système à un instant, $d t$, après l'excitation. En variant le retard, $d t$, entre ces deux impulsions, il est possible de suivre la dynamique des matériaux avec une résolution meilleure que $100 \mathrm{fs}[3,4]$.

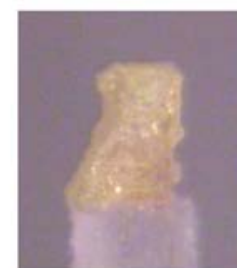

L
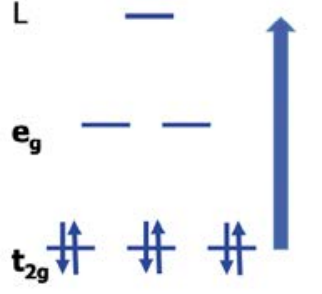

\section{Diamagnetique \\ Bas Spin \\ ${ }^{1} \mathrm{~A}_{1} \mathrm{~S}=\mathbf{0}\left(t_{2 g}{ }^{6} e_{g}{ }^{9}\right)$}
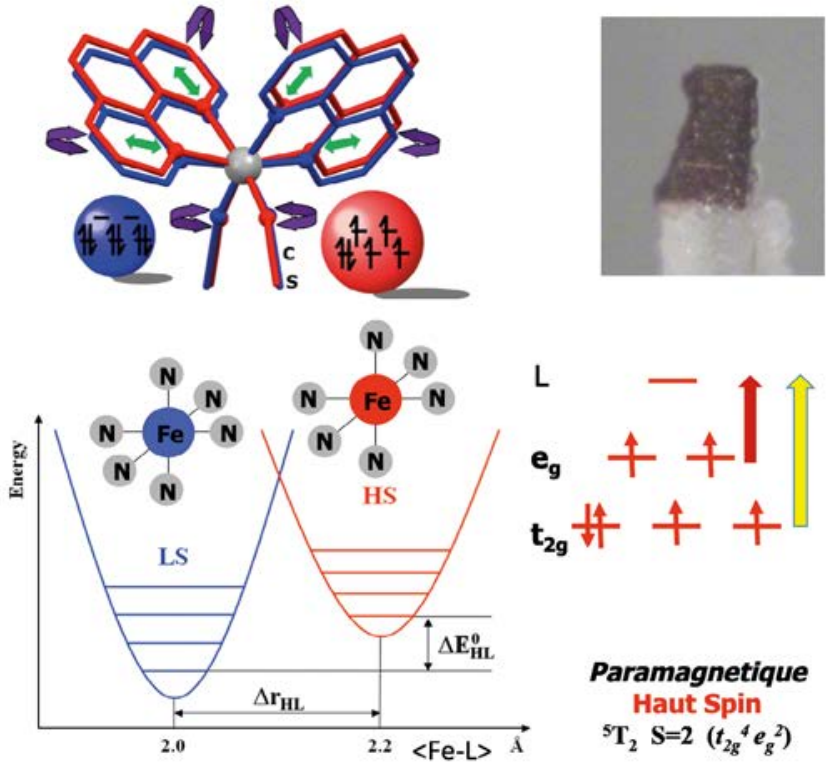

Figure 1. Changement de couleur d'un cristal à transition de spin et représentation schématique des structures moléculaires et électroniques et des transitions optiques (flèches). Les deux états de spin (LS et HS) ont deux structures moléculaires différentes, en particulier au niveau des longueurs de liaison Fe-L.

Si la sonde est un laser visible, disponible au laboratoire, il est possible de voir le changement d'état magnétique au travers du changement de couleur associé (Fig. 2b). Malheureusement, les informations sur les réorganisations électroniques et structurales obtenues par spectroscopie optique sont relativement indirectes. L'émergence de nouvelles sources de rayonnement ultra-bref dans le domaine des rayons $\mathrm{X}$ donne un accès plus direct à ces dynamiques. Les lasers à électrons libres dans le domaine des rayons X (X-FEL, Fig. 2c) sont des sources de rayonnement de quatrième génération, qui accélèrent des électrons sur plusieurs km de long à une vitesse proche de celle de la lumière pour les faire laser dans le domaine des rayons X. Ces X-FEL génèrent un rayonnement $\mathrm{X}$ laser de brillance phénoménale, avec des durées d'impulsions de quelques fs [6]. Il est alors possible des réaliser des expériences pompe optique - sonde $\mathrm{X}$ avec une résolution de $\sim 25$ fs. Différentes

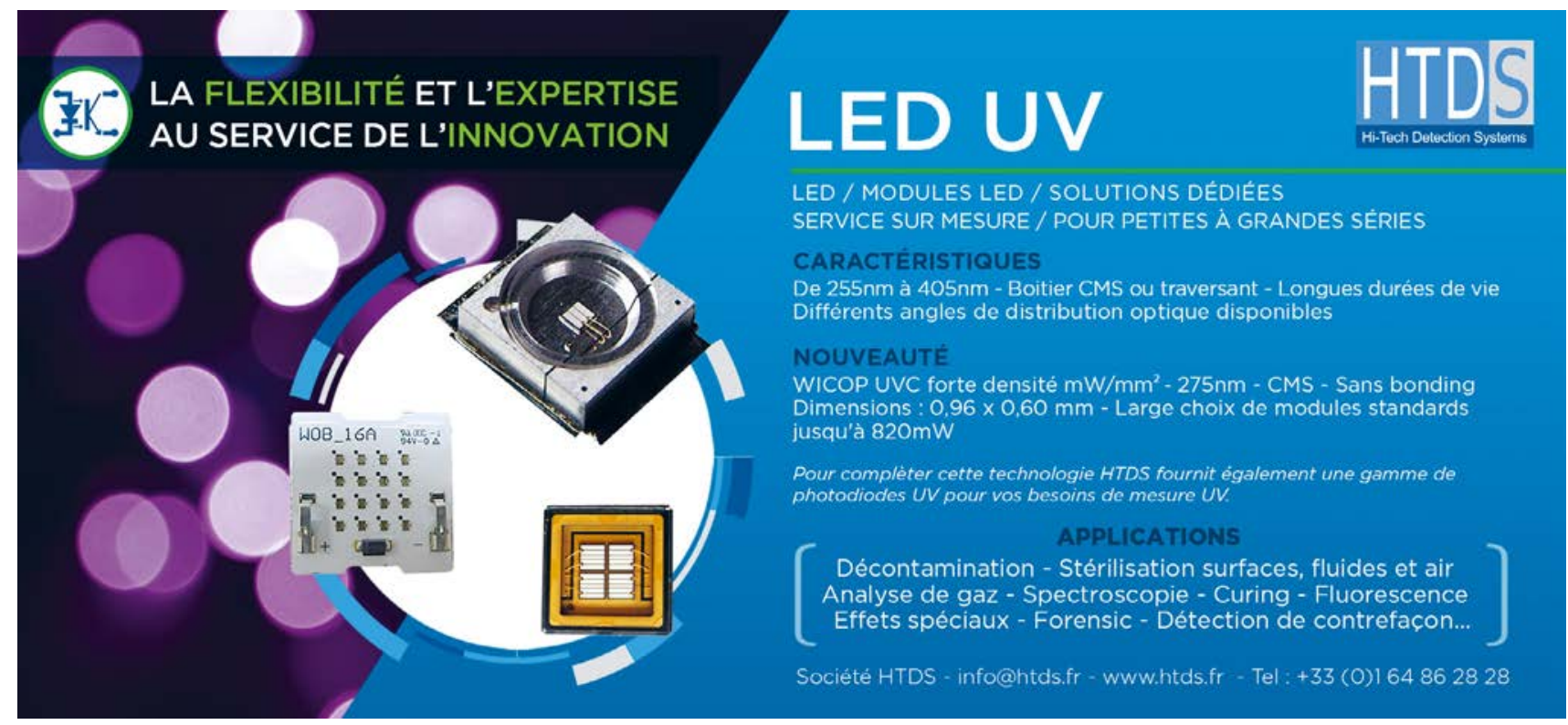


a)

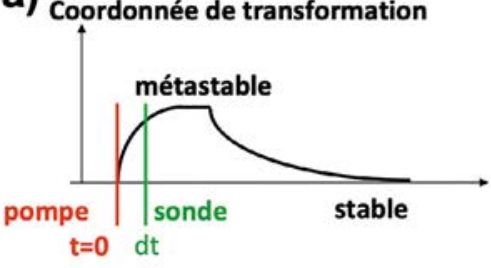

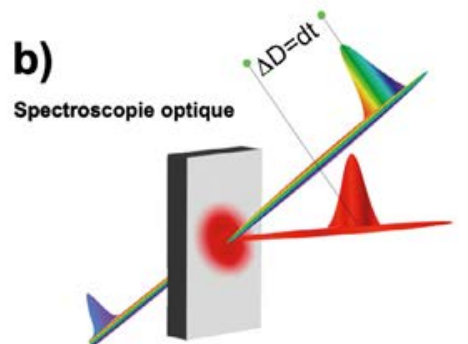

c)

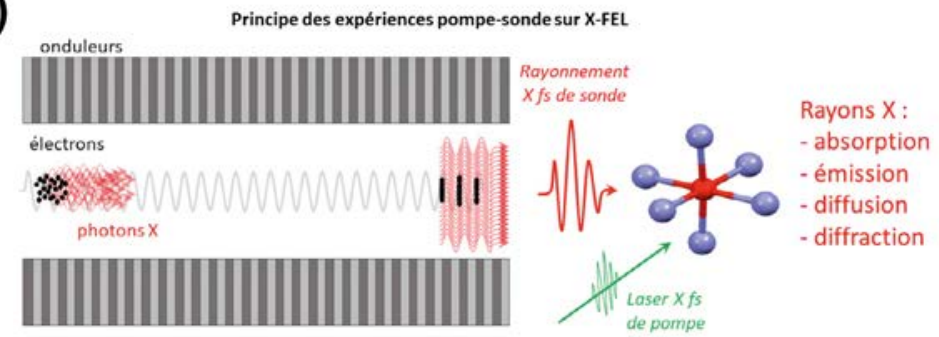

techniques d'absorption, d'émission, de diffusion ou de diffraction des rayons $\mathrm{X}$ apportent des informations complémentaires sur les structures électroniques et moléculaires de la matière [6]. Il est essentiel de combiner ces expériences optiques au laboratoire et rayon X sur X-FEL pour comprendre les dynamiques photoinduites des différents degrés de liberté mis en jeu. Suivre leur évolution hors équilibre et leurs interactions permet de comprendre l'émergence de fonctions et de les contrôler [3,4].

\section{CONVERSION D'ÉTAT}

\section{DE SPIN ULTRA-RAPIDE}

Les matériaux moléculaires bistables présentant l'effet LIESST (Fig. 1) possèdent deux états de spins différents: l'état LS de configuration électronique $\mathrm{S}=0\left(\mathrm{t}_{2 \mathrm{~g}}{ }^{6} \mathrm{e}_{\mathrm{g}}{ }^{0} \mathrm{~L}^{0}\right)$ et l'état $\mathrm{HS}\left(\mathrm{S}=2, \mathrm{t}_{2 \mathrm{~g}}{ }^{4} \mathrm{e}_{\mathrm{g}} \mathrm{L}^{0}\right)$. L'effet LIESST consiste à exciter par la lumière un système dans l'état LS pour le faire basculer vers l'état HS. Ce phénomène se produit à léchelle de temps des mouvements atomiques élémentaires et implique une réorganisation de la structure moléculaire, liée à la variation de la longueur de liaison Fe-L, qui accompagne le peuplement d'états anti-liants $e_{g}$. En utilisant la spectroscopie d'absorption au X-FEL LCLS à Stanford [4], avec une résolution temporelle de $\sim 25 \mathrm{fs}$, nous avons pu observer de façon directe la dynamique structurale cohérente du ligand lors de l'effet LIESST par des mesures XANES au seuil du Fe (Fig.3). Ces données expérimentales

permettent de séparer le changement moyen d'état électronique et la dynamique structurale. L'excitation optique $\mathrm{t}_{2 \mathrm{~g}} \rightarrow \mathrm{L}$ induit un état de transfert de charge métal-ligand (MLCT $\mathrm{t}_{2 \mathrm{~g}}{ }^{5} \mathrm{e}_{\mathrm{g}} \mathrm{L}^{1}$ ), et ce changement de $\mathrm{Fe}^{2+} \grave{\text { à }} e^{3+}$ diminue l'absorption des rayons $\mathrm{X}$. Cet état relaxe ensuite vers l'état HS via l'activation et l'amortissement du mode de respiration de la molécule lié à l'allongement de la distance Fe-L, et qui est caractérisé par une augmentation et une oscillation de l'absorption des rayonsX.Ilestalorspossible de décomposer les dynamiques électroniques et structurales comme montré sur la figure 3. Nos études plus récentes ont montré qu'il existe différents chemins possibles de transformation et que l'effet LIESST est plus rapide sous excitation $\mathrm{t}_{2 \mathrm{~g}} \rightarrow \mathrm{e}_{\mathrm{g}}$ que sous excitation $\mathrm{t}_{2 \mathrm{~g}} \rightarrow \mathrm{L}$ [7]. La longueur d'onde d'excitation devient alors un paramètre de contrôle de la vitesse et de l'efficacité du processus.
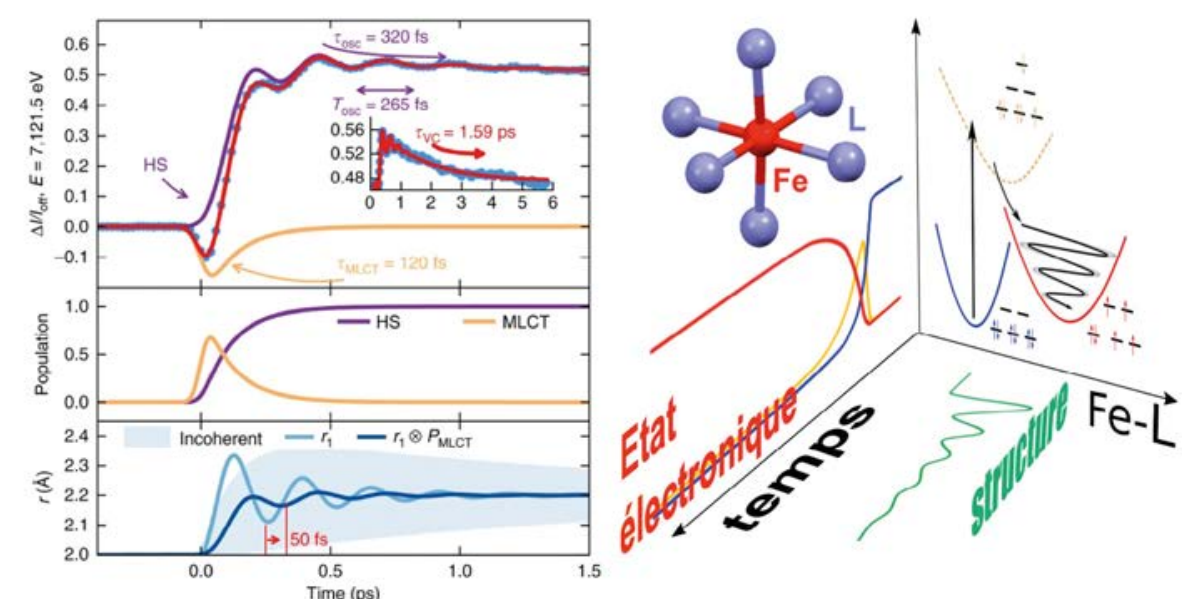

Figure 2. Principe des expériences pompe-sonde. (a) L'état du système est sondé à un instant $d t$ après l'excitation par le laser de pompe. (b) Spectroscopie optique où la sonde est une impulsion fs de lumière visible. (c) Expériences pompe-sonde sur X-FEL où la sonde est une impulsion laser de rayons $X$.

\section{TRANSFERT DE CHARGE ET MAGNÉTISME}

Nous avons aussi étudié des analogues dubleu de Prusse qui sont des systèmes photomagnétiques [4]. Ce sont des réseaux de coordination où les groupes cyanures relient les ions Co et Fe dans les champs cristallins octaédriques. Il existe deux configurations électroniques - structurales bistables (Fig. 4) liées à un transfert de charge (CT) entre le Fe et le Co : $\mathrm{Co}{ }^{\| l}(\mathrm{~S}=0) \mathrm{Fe}^{\|}(\mathrm{S}=0)$ ou $\mathrm{Co}^{\|}(\mathrm{S}=3 / 2) \mathrm{Fe}^{\|\|}(\mathrm{S}=1 / 2)$. Dans l'état HS Coll Felll, la population des orbitales $\mathrm{Co}\left(\mathrm{e}_{\mathrm{g}}\right)$ anti-liantes allonge les liaisons Co-N de $\approx 0,2 \AA$ et cette transition de spin (ST) sur le Co est la principale coordonnée de réaction. Depuis 1996 où Sato a mis en évidence ce transfert de charge photo-induit $\mathrm{Fe} \rightarrow \mathrm{Co}$, les mécanismes de ce processus sont restés inaccessibles et il était alors considéré que la voie suivie lors de la transformation est un transfert de

Figure 3. Évolution du signal d'absorption des rayons $X$ (gauche) d'un système à transition de spin après excitation laser optique fs [4]. Le signal se décompose en termes de changement d'état électronique et de dynamique structurale avec l'évolution de la distance rentre le Fe et le ligand (droite). 

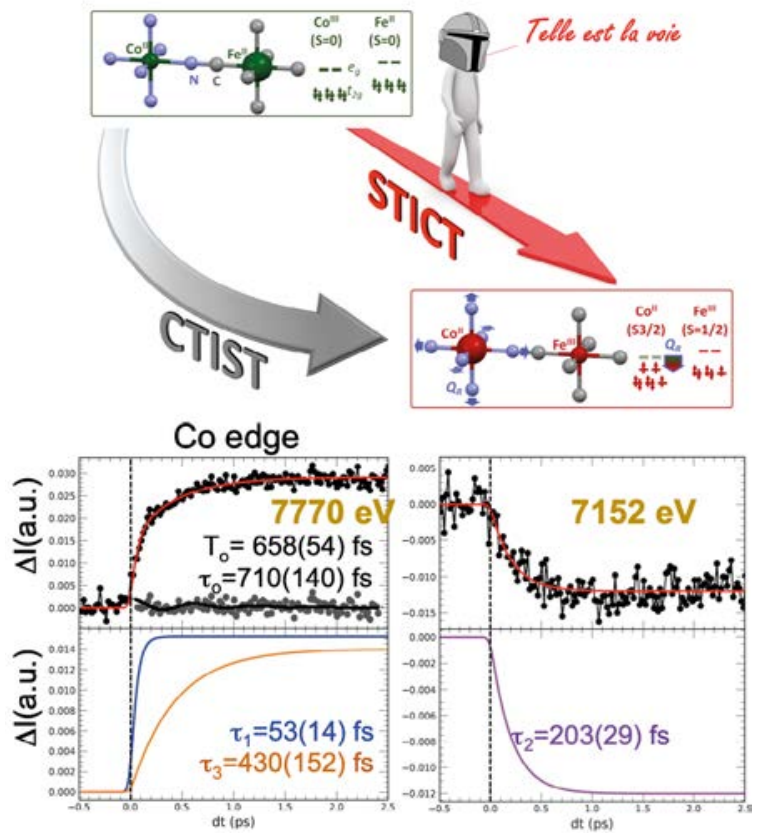

Figure 4. Transition

photomagnétique entre états bas et haut spin.

Les mesures de spectroscopie XANES montrent que c'est la transition de spin photoinduite (ST) en 50 fs autour du Co qui induit le transfert de charge (CT) observé en 200 fs au seuil du Fe, suivant ainsi le chemin de transformation STICT.

charge photoinduit qui induit à son tour la transition de spin (voie de transformation CTIST). Nous avons utiliséles mesures XANES résolues en temps au X-FEL LCLS ( $\approx 25 \mathrm{fs}$ ) pour sonder la dynamique des degrés de liberté électroniques et structuraux [4]. Nos résultats donnent un aperçu direct des dynamiques électroniques et structurales. L'excitation optique de type Coll $\left(\mathrm{t}_{2 \mathrm{~g}}\right) \rightarrow \mathrm{Co}^{\| l}\left(\mathrm{e}_{\mathrm{g}}\right)$ induit la transition de spin sur le Co (allongeant les liaisons Co-N en $\approx 50 \mathrm{fs}$ ), caractérisé par une variation rapide de l'absorption au-dessus du seuil du Co (7770 eV, Fig. 4). Le XANES est une technique de choix pour mesurer les changements d'état d'oxydation des métaux, et nous avons mis en évidence que le transfert de charge $\mathrm{Fe} \rightarrow \mathrm{Co}(\mathrm{CT})$ n'a lieu que dans une seconde étape en $\approx 200 \mathrm{fs}$, comme observé au seuil du Fe (7152 eV). Ces résultats apportent une réponse claire à une question débattue depuis des décennies, en démontrant que dans ce composé CoFe prototype photoexcité, la voie de transformation est une transition de spin photoactivée qui induit le transfert de charge (STICT sur la Fig. 4).

\section{CONCLUSION}

Cet axe de recherche autour de la science ultra-rapide des transformations des matériaux est en plein essor, avec des techniques permettant de suivre et de contrôler les mécanismes électroniques et atomiques responsables des modifications de fonctions de matériaux. Des axes de recherche émergeant portent à présent sur des transformations induites par des rayonnements de plus basse énergie (IR et $\mathrm{THz}$ ) qui peuvent représenter de nouvelles opportunités pour de futures technologies photoniques basées sur les modifications de propriétés des matériaux. Ces processus photoinduits pourraient être intégrés à plus long terme dans divers dispositifs (mémoire, capteurs...).

\section{RÉFÉRENCES}

[1] E. Collet et al, Science 300, 612-615 (2003)

[2] G. Azzolina et al, Eur. J. Inorg. Chem. 27, 3142-3147 (2019)

[3] M. Cammarata et al, Nature Chemistry 13, 10-14 (2021)

[4] H. T. Lemke et al, Nat. Commun. 8, 15342 (2017)

[5] N. Konstantinov et al, J. Mater. Chem. C 9, 2712-2720 (2021)

[6] M. Chergui and E. Collet, Chem. Rev. 117, 11025-11065 (2017)

[7] S. Zerdane et al, Chem. Sci. 8, 4978-4986 (2017) 